\title{
Electrocatalysis and electron transfer mechanisms in the reduction of organic halides at Ag
}

\author{
Abdirisak Ahmed Isse, ${ }^{1}$ Giacomo Berzi, ${ }^{2}$ Luigi Falciola, ${ }^{2}$ Manuela Rossi, ${ }^{2}$ Patrizia R. Mussini, ${ }^{2 *}$ \\ $\underline{\text { Armando Gennaro, }}{ }^{1}$ * \\ ${ }^{1}$ Dep. of Chemical Sciences, University of Padova, via Marzolo 1, 35131 Padova \\ ${ }^{2}$ Dep. of Physical Chemistry and Electrochemistry, University of Milano, via Golgi 19, 20133 Milano
}

\begin{abstract}
The reductive cleavage of a series of organic halides, including both aromatic and aliphatic compounds, has been investigated in acetonitrile at glassy carbon and silver electrodes. Ag exhibits extraordinary electrocatalytic activities for the reduction of most of the investigated halides. During the reductive cleavage of a carbon-halogen bond, electron transfer (ET) and bond breaking may occur either in a single step or in two distinct steps. The compounds examined in this study are representative of both dissociative electron transfer (DET) mechanisms. In general a link between the DET mechanism and electrocatalysis at Ag is observed for the whole set of data. There is no catalysis at all when the ET involves a substituent that gives a stable radical anion. Furthermore, there is no catalysis for all aromatic chlorides. Instead, a remarkable electrocatalysis is observed for all compounds undergoing a concerted DET mechanism, regardless of the nature of the halogen atom.
\end{abstract}

Keywords: Electrocatalysis, silver electrode, dissociative electron transfer, organic halides. 


\section{Introduction}

The electrochemical reductive cleavage of carbon-halogen bonds in organic compounds has been the object of numerous studies and several reviews are available [1-3]. The great interest for this process is due to the important roles it plays in synthetic [2,4] and environmental applications, [57] especially the abatement of volatile polychlorinated organic compounds, but also to mechanistic aspects.

It is well known that the injection of one electron into an organic halide, by reaction with homogeneous electron donors as well as by electrochemical or other means, leads to the fragmentation of the carbon-halogen $\sigma$ bond. There are two possible reaction mechanisms for such reductive cleavages: electron transfer (ET) and bond breaking can occur either in a stepwise manner, with the intermediate formation of a radical anion (eqs. 1, 2), or in a concerted way in which the two fragments, the organic radical and the halide leaving ion, are produced in a single step (eq. 3).

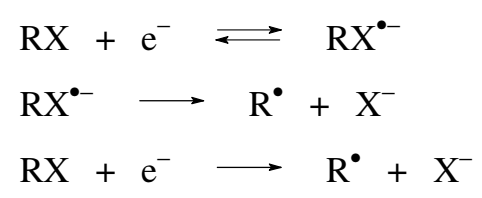

Reduction of organic halides at inert electrodes or by electrogenerated homogeneous electron donors has been widely investigated to test the dissociative electron transfer (DET) theory developed by Savéant [8] for the concerted mechanism. While such mechanistic investigations rigorously require the use of outer sphere electron donors, catalytic electrode surfaces are highly demanded in preparative scale processes. The radical $\mathrm{R}^{\bullet}$ may undergo typical radical reactions or may be reduced to a carbanion (eq. 4), its reduction potential being generally more positive than that of the halide, giving very useful following reactions.

$$
\mathrm{R}^{\bullet}+\mathrm{e}^{-} \longrightarrow \mathrm{R}^{-}
$$


A major drawback of the electrochemical activation of carbon-halogen bonds is that the process requires very negative electrode potentials. Thus, much attention has been devoted to develop electrocatalytic processes, either by utilizing homogeneous redox catalysts, such as metal complexes or aromatic mediators, or by the use of electrocatalytic cathodic materials. We have been active in the field of electrocatalytic activation of organic halides for several years. In particular, we focused our attention on silver [9-14], which is among the best catalytic materials for the reduction of RX [15].

The electrocatalytic properties of silver have been exploited in several electrosynthetic processes, in particular the electrosynthesis of fine chemicals and pharmaceutical products by electrocarboxylation of the corresponding chlorides $[16,17]$. In some cases, also a first scale up has been developed with very encouraging results [18].

The mechanism of the electrocatalytic process at $\mathrm{Ag}$ has been investigated from different viewpoints. It has been shown that several factors play an important role in the electrocatalytic process; these include the molecular structure of the organic halide, the nature of the halogen atom, the surface morphology of the electrode, the adsorption of the reagents and products, especially halide ions and also the solvent [19].

Recently we have found that also the mechanism of the dissociative electron transfer has a crucial role in the electrocatalytic process [20]. We have investigated the electrochemical reduction of a series of chlorides, representative of both stepwise and concerted DET mechanisms, at Ag. This study has shown a clear link between electrocatalysis and DET mechanism. In fact, we have found that for all chlorides undergoing a stepwise DET no electrocatalytic activity is exhibited by Ag, whereas for all of the chlorides undergoing a concerted DET, Ag exhibits an impressive electrocatalytic activity; in the latter case, the reduction potentials at $\mathrm{Ag}$ are shifted to more positive values by several hundreds of millivolts with respect to a non catalytic electrode such as glassy carbon (GC). 
We have extended this investigation to other cathodic materials with the aim of verifying if this finding is peculiar of $\mathrm{Ag}$ or is a more general one [21]. In such study, the electroreduction of various organic chlorides, as before, representative of both stepwise and concerted DET mechanisms, was comparatively analysed at GC and at some potential electrocatalytic cathodes, namely Ag, Ag nanoclusters electrodeposited on GC [22], $\mathrm{Cu}$ [23] and Pd [24]. These metals as well as their Ag-Pd [25] and Cu-Pd [26] alloys have been reported to be good electrocatalytic materials for the reduction of several alkyl iodides. For all of the investigated cathodic materials, we have obtained a clear mechanistic discrimination: no electrocatalytic activity was observed for all those compounds undergoing a stepwise DET, whereas remarkable electrocatalytic activities of comparable strength were displayed by all of the cathodic materials when the process occurred by a concerted DET mechanism. In the latter case, impressive positive shifts of the reduction potentials of up to $800 \mathrm{mV}$ were observed for the catalytic electrodes as compared to GC.

The correlation between the electrocatalytic activity and the mechanism of DET gives new insights on the comprehension of electrocatalysis. While this correlation appears to be very clear in the reduction of organic chlorides, less information is available for bromides and iodides. With the aim of studying in depth the role of DET mechanism in the electrocatalytic activity of cathodic materials, we have investigated the reduction of a series of organic halides at silver. The investigated halides are depicted in Scheme 1 and were selected to be representative of both stepwise and concerted DET mechanisms as well as all types of halogen atoms. We have compared the electrochemical behaviour at $\mathrm{Ag}$ with that at $\mathrm{GC}$, by analysing the voltammetric responses in $\mathrm{CH}_{3} \mathrm{CN}$. 


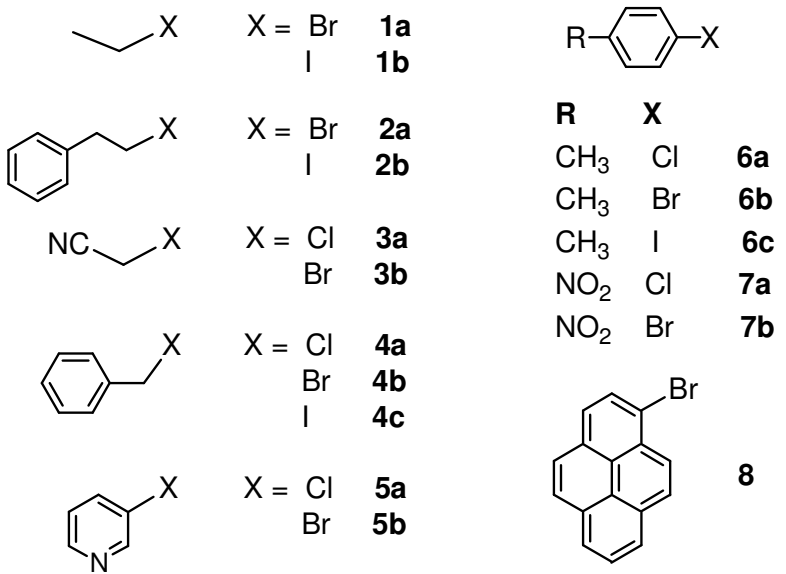

Scheme 1

\section{Experimental}

Acetonitrile (Merck, HPLC grade) and the supporting electrolyte $\mathrm{Et}_{4} \mathrm{NBF}_{4}$ (Fluka, puriss) were used as received. The organic halides (scheme 1) were purchased from Sigma-Aldrich and were used without further purification.

Electrochemical measurements were performed in a three electrode glass cell, using a computer controlled potentiostat (AutoLab PGSTAT30, Eco Chemie, The Netherlands). The working electrodes were glassy carbon (GC) and Ag, while the counter and reference electrodes were a Pt coil and a saturated calomel electrode (SCE), respectively. The polycrystalline silver electrodes were prepared by electrodeposition of $\mathrm{Ag}$ on a Pt wire (diameter: $0.05 \mathrm{~cm}$, length: $1 \mathrm{~cm}$ ) from a 10 $\mathrm{g} \mathrm{dm}^{-3} \mathrm{KAg}(\mathrm{CN})_{2}$ bath at a current density $i=0.5 \mathrm{mAcm}^{-2}$ for $15-18 \mathrm{~h}$, using a silver foil as anode. The electrode was then carefully washed with deionized water followed by 5 min sonication in acetonitrile in a Bransonic 221 ultrasound bath. This procedure affords reproducible, controlled surfaces [27] and thus a freshly prepared electrode was used in each experiment. The GC electrode (AMEL, $3 \mathrm{~mm}$ diameter disk) was polished with a wet diamond powder (Aldrich, $1 \mu \mathrm{m}$ ) on a 
polishing cloth (Struers, DP-Nap) and ultrasonically rinsed in $\mathrm{CH}_{3} \mathrm{CN}$ for 5 minutes. All measurements were carried out at $298 \mathrm{~K}$ and the solutions were deoxygenated with pure nitrogen prior to use.

\section{Results}

The relevant voltammetric data pertaining to all of the investigated halides are reported in Tables 1 and 2 for GC and Ag electrodes, respectively. Besides peak potentials, we report the results of voltammetric analyses, in particular the values of the electron transfer coefficient $\alpha$, which have been estimated, as a first approximation, both from the peak potential slope $\partial E_{\mathrm{p}} / \partial \log v$ and from the peak width $E_{\mathrm{p} / 2}-E_{\mathrm{p}}$, according to the equations $\partial E_{\mathrm{p}} / \partial \log v=-1.15 R T / F \alpha$ and $E_{\mathrm{p} / 2}-E_{\mathrm{p}}=1.857 R T / F \alpha$, which are valid for an irreversible ET [28]. In Table 2, also the catalytic activity of Ag, which may be quantified by $\Delta E_{\mathrm{p}}=E_{\mathrm{p}}{ }^{(\mathrm{Ag})}-E_{\mathrm{p}}^{(\mathrm{GC})}$, is reported in the last column.

Assuming that GC is a non catalytic material, the ET may be considered as an outer sphere process, at least from the viewpoint of the donor. Thus, in the case of a stepwise mechanism, we expect the electron transfer coefficient $\alpha$ to be around or greater than 0.5 . This is, in fact, the case for halides $\mathbf{5 a}, \mathbf{b}, \mathbf{6 a}, \mathbf{b}, \mathbf{c}$ and $\mathbf{8}$. In the case of the nitro derivatives $\mathbf{7 a}, \mathbf{b}$, use of the above mentioned equations is not permissible because ET to these compounds is both chemically and electrochemically reversible.

The values of $\alpha$ calculated by the same equations for the ET at Ag are less indicative than in the case of GC, owing to the possible involvement of silver surface in the ET, especially in the case of bromides and iodides. In particular, the presence of adsorption phenomena modifies the shape of the peaks, making calculation of $\alpha$ from the peak width quite unreliable. The results obtained by the 
two equations are often comparable (Table 2), though significant differences can be observed in some cases. Since, unlike the peak width, $\partial E_{\mathrm{p}} / \partial \log v$ is little influenced by adsorption phenomena, data obtained from the slope can be used for mechanistic considerations.
Formattato: Inglese (Gran Bretagna)

Formattato: Inglese (Gran

Bretagna)

Table 1. Data for the electrochemical reduction of $\mathrm{RX}$ in $\mathrm{CH}_{3} \mathrm{CN}+0.1 \mathrm{M} \mathrm{Et}_{4} \mathrm{NBF}_{4}$ at GC.

\begin{tabular}{|c|c|c|c|c|c|c|}
\hline $\mathrm{RX}$ & $\begin{array}{l}E_{\mathrm{p}}^{\mathrm{a}} \\
/ \mathrm{V}\end{array}$ & $\begin{array}{l}-\partial E_{\mathrm{p}} / \partial \log v \\
/ \mathrm{V}\end{array}$ & $\alpha^{\mathrm{b}}$ & $\begin{array}{l}E_{\mathrm{p} / 2}-E_{\mathrm{p}} \\
/ \mathrm{V}\end{array}$ & $\alpha^{\mathrm{c}}$ & $\begin{array}{l}\text { DET } \\
\text { mechanism }^{\mathrm{d}}\end{array}$ \\
\hline $1 \mathrm{a}$ & -2.57 & & & 0.170 & 0.28 & $\mathrm{c}$ \\
\hline $1 b$ & -2.29 & 0.074 & 0.40 & 0.144 & 0.33 & $\mathrm{c}$ \\
\hline $2 a$ & -2.37 & & & 0.140 & 0.34 & $\mathrm{c}$ \\
\hline $2 b$ & -2.03 & 0.068 & 0.44 & 0.121 & 0.39 & $\mathrm{c}$ \\
\hline $3 \mathbf{a}^{\mathrm{e}}$ & -2.04 & 0.103 & 0.29 & 0.145 & 0.33 & $\mathrm{c}$ \\
\hline $3 b$ & -1.55 & 0.092 & 0.32 & 0.156 & 0.31 & $\mathrm{c}$ \\
\hline $4 a^{f}$ & -2.25 & 0.103 & 0.29 & 0.159 & 0.30 & $\mathrm{c}$ \\
\hline $4 b^{f}$ & -1.80 & 0.090 & 0.33 & 0.138 & 0.35 & $\mathrm{c}$ \\
\hline $4 c^{f}$ & -1.46 & 0.095 & 0.31 & 0.116 & 0.41 & $\mathrm{c}$ \\
\hline $5 a^{e}$ & -2.30 & 0.047 & 0.63 & 0.064 & 0.75 & sw \\
\hline $5 b$ & -2.15 & 0.056 & 0.53 & 0.073 & 0.65 & sw \\
\hline $6 a$ & -2.77 & 0.057 & 0.52 & 0.075 & 0.64 & sw \\
\hline $6 b$ & -2.58 & 0.071 & 0.42 & 0.113 & 0.42 & sw \\
\hline $6 c$ & -2.12 & 0.074 & 0.40 & 0.104 & 0.46 & sw \\
\hline $7 \mathbf{a}$ & -1.09 & 0 & & 0.058 & & sw \\
\hline $7 b$ & -1.06 & 0 & & 0.057 & & sw \\
\hline 8 & -1.69 & 0.041 & 0.72 & 0.060 & 0.80 & sw \\
\hline
\end{tabular}

${ }^{\mathrm{c} C a l c u l a t e d}$ from $E_{\mathrm{p} / 2}-E_{\mathrm{p}}=1.857 R T / F \alpha .{ }^{\mathrm{d}} \mathrm{DET}$ mechanism: concerted (c) or stepwise (sw).

${ }^{\mathrm{e}}$ Taken from ref [21]. ${ }^{\mathrm{f}}$ Taken from ref [13]. 
Table 2. Data for the electrochemical reduction of $\mathrm{RX}$ in $\mathrm{CH}_{3} \mathrm{CN}+0.1 \mathrm{M} \mathrm{Et}_{4} \mathrm{NBF}_{4}$ at $\mathrm{Ag}$.

\begin{tabular}{|c|c|c|c|c|c|c|c|}
\hline $\mathrm{RX}$ & $\begin{array}{l}E_{\mathrm{p}}{ }^{\mathrm{a}} \\
/ \mathrm{V}\end{array}$ & $\begin{array}{l}-\partial E_{\mathrm{p}} / \partial \log v \\
/ \mathrm{V}\end{array}$ & $\alpha^{\mathrm{b}}$ & $\begin{array}{l}E_{\mathrm{p} / 2}-E_{\mathrm{p} /} \\
/ \mathrm{V}\end{array}$ & $\alpha^{c}$ & $\begin{array}{l}\text { DET } \\
\text { mechanism }^{\text {d }}\end{array}$ & $\begin{array}{l}\Delta E_{\mathrm{p}}^{\mathrm{e}} \\
/ \mathrm{V}\end{array}$ \\
\hline $1 a$ & -1.60 & 0.101 & 0.29 & 0.167 & 0.29 & $\mathrm{c}$ & 0.97 \\
\hline $1 b$ & -1.23 & 0.071 & 0.42 & 0.056 & 0.85 & $\mathrm{c}$ & 1.06 \\
\hline $2 a$ & -1.58 & 0.095 & 0.31 & 0.133 & 0.36 & $\mathrm{c}$ & 0.79 \\
\hline $2 b$ & -1.45 & 0.083 & 0.36 & 0.086 & 0.55 & $\mathrm{c}$ & 0.58 \\
\hline $3 \mathbf{a}^{\mathrm{f}}$ & -1.32 & 0.090 & 0.33 & 0.130 & 0.37 & $\mathrm{c}$ & 0.72 \\
\hline $3 b$ & -0.97 & 0.193 & 0.15 & 0.192 & 0.25 & $\mathrm{c}$ & 0.59 \\
\hline $4 a^{g}$ & -1.79 & 0.102 & 0.29 & 0.141 & 0.34 & $\mathrm{c}$ & 0.46 \\
\hline $4 b^{g}$ & -1.08 & 0.113 & 0.26 & & & $\mathrm{c}$ & 0.72 \\
\hline $4 c^{g}$ & -0.98 & 0.137 & 0.22 & 0.212 & 0.23 & $\mathrm{c}$ & 0.48 \\
\hline $5 a^{h}$ & -2.28 & 0.040 & 0.74 & 0.063 & 0.76 & sw & 0.02 \\
\hline $5 b$ & -1.55 & 0.099 & 0.30 & 0.082 & 0.58 & sw & 0.61 \\
\hline $6 a$ & $\leq-2.8$ & & & & & sw & \\
\hline $6 b$ & -1.75 & 0.106 & 0.28 & 0.188 & 0.25 & sw & 0.83 \\
\hline $6 c$ & -1.23 & 0.071 & 0.42 & 0.103 & 0.46 & sw & 0.89 \\
\hline $7 \mathbf{a}$ & -1.09 & 0 & & 0.060 & & sw & 0 \\
\hline $7 b$ & -1.06 & 0 & & 0.061 & & sw & 0 \\
\hline 8 & -1.41 & 0.096 & 0.31 & 0.105 & 0.45 & sw & 0.28 \\
\hline
\end{tabular}

${ }^{\mathrm{c}}$ Calculated from $E_{\mathrm{p} / 2}-E_{\mathrm{p}}=1.857 R T / F \alpha .{ }^{\mathrm{d}} \mathrm{DET}$ mechanism at GC: concerted (c) or stepwise (sw). ${ }^{\mathrm{e}} \Delta E_{\mathrm{p}}=E_{\mathrm{p}}{ }^{(\mathrm{Ag})}-E_{\mathrm{p}}{ }^{(\mathrm{GC})}\left(E_{\mathrm{p}}{ }^{(\mathrm{Ag})}\right.$ and $E_{\mathrm{p}}{ }^{(\mathrm{GC})}$ are the peak potentials measured at $\mathrm{Ag}$ and $\mathrm{GC}$ cathodes, respectively). ${ }^{\mathrm{f}}$ Taken from ref [21]. ${ }^{\mathrm{g}}$ Taken from ref [13]. ${ }^{\mathrm{h}}$ Taken from ref [20].

\subsection{Chlorides}

The investigated chlorides are representative of both concerted (3a, 4a) and stepwise (5a, 6a, 7a) DET mechanisms. It is well recognised that aromatic chlorides undergo a stepwise DET (eqs 1$3)$, thanks to their low lying $\pi^{*}$ orbitals in which the incoming electron is accommodated. 
Moreover, since the aromatic radical produced by the rupture of the bond, is more easily reducible than the starting halide, often the first reduction peak corresponds to a two electron process leading to the formation of the chloride ion and a carbanion, which is protonated by any proton donor HA present in solution (eq. 5) [29, 30].

$$
\mathrm{R}^{-}+\mathrm{HA} \longrightarrow \mathrm{RH}+\mathrm{A}^{-}
$$

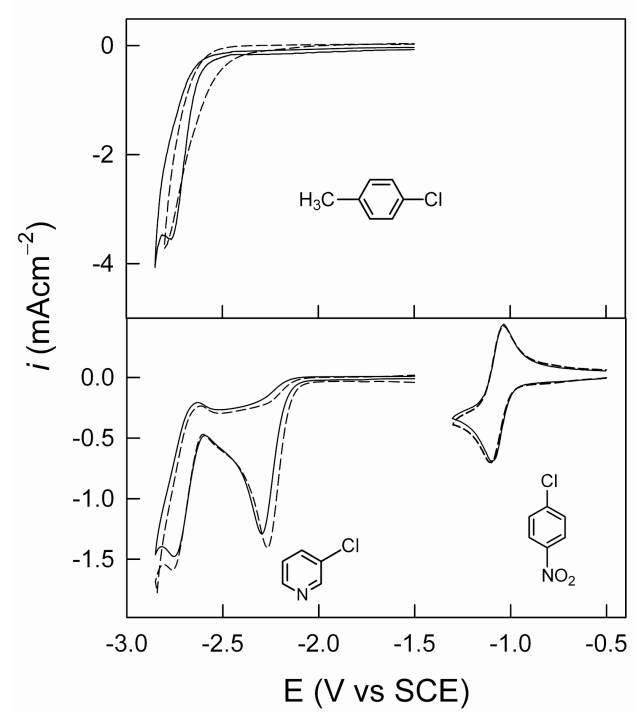

Fig. 1. Cyclic voltammetry of 4-chlorotoluene $\mathbf{6 a}(4 \mathrm{mM})$, 4-chloronitrobenzene $7 \mathbf{a}(0.79 \mathrm{mM})$ and 3-chloropyridine $(1.05 \mathrm{mM})$ in $\mathrm{CH}_{3} \mathrm{CN}+0.1 \mathrm{M} \mathrm{Et}_{4} \mathrm{NBF}_{4}$ recorded at $0.2 \mathrm{Vs}^{-1}$ at $\mathrm{GC}$ (full lines) and $\mathrm{Ag}$ (dashed lines).

If the aromatic hydrocarbon $\mathrm{RH}$ is reducible, as, for example, in the case of chloropyridine (see Fig. 1), the voltammetric response relative to its reduction is always present and does not appreciably depend on the nature of the electrode material.

The voltammetric responses at GC are typical of a stepwise DET: $\alpha$ is greater than 0.5 in the case of chloropyridine, both at GC and Ag. Also for chlorotoluene $\alpha$ is greater than 0.5 , but in this 
case we have only the value at GC. In fact, at Ag the solvent-electrolyte discharge starts at about $-2.55 \mathrm{~V}$ so that the reduction peak of chlorotoluene is completely covered. The voltammogram reported in Fig. 1 was obtained by subtracting a blank cyclic voltammogram from that recorded for a solution of the substrate. Although a well defined peak is not obtained even after this procedure, this experiment allows a rough estimation of the reduction potential at Ag, which, as shown, is quite similar to that at GC. This means that no electrocatalytic effect is displayed by Ag for the reduction of this substrate.

The behaviour of 4-nitrochlorobenzene 7a is quite different from the other aromatic chlorides. It shows a reversible peak couple (Fig. 1) followed by several reduction peaks (not shown) and associated anodic peaks. The first reduction peak corresponds to a reversible one electron transfer, which is typical of nitrocompounds [31,32]. It is worth noting that the behaviour on GC and Ag is practically the same: no electrocatalysis is displayed by Ag, as expected for a stepwise DET, but also there is no influence on the reversibility of the first ET, which is completely centred on the nitro group. These results confirm the general finding that, in the case of a reversible (i.e. fast) ET, both Ag [20] and other electrocatalytic cathodic materials [21] act simply as outer sphere electron donors.

For the chlorides 3a and 4a, which undergo a concerted DET, $\alpha$ measured at GC is significantly lower than 0.5 . In effect, concerted DETs generally exhibit values of $\alpha<0.35$, since the high overpotential required to overcome the high activation energy mainly due to the breaking bond, shifts the reduction peak to potentials considerably more negative than the standard potential and thus, according to Marcus-Hush ET theory, $\alpha$ is greatly diminished. Quite low values of $\alpha$ have been obtained also at $\mathrm{Ag}$, indicating that also at this electrode the reductive cleavage of $\mathbf{3 a}$ and $\mathbf{4 a}$ follows a concerted mechanism. The most relevant result of this comparative analysis is that, for these chlorides, Ag exhibits a remarkable electrocatalytic activity and, as shown in Table 2, the reduction peak at $\mathrm{Ag}$ is shifted to more positive potentials with respect to GC; the observed positive shifts are $\Delta E_{\mathrm{p}}=0.72$ and $0.46 \mathrm{~V}$ for $\mathbf{3 a}$ and $\mathbf{4 a}$, respectively. 


\subsection{Bromides}

Some representative examples of the voltammetric patterns of the bromides, which constitute the most extensive of the examined series, are illustrated in Figs. 2 and 3. In most cases, the first reduction peak represents an overall $2 \mathrm{e}^{-}$process leading to the hydrodehalogention of the substrate. When the newly formed compound $\mathrm{RH}$ is reducible before the cathodic solvent-electrolyte discharge, further reduction peak or peaks, which are independent of the nature of the electrode material, are observed; we report as an example the complete cyclic voltammogram of 1-bromopyrene 8 (Fig. 2).

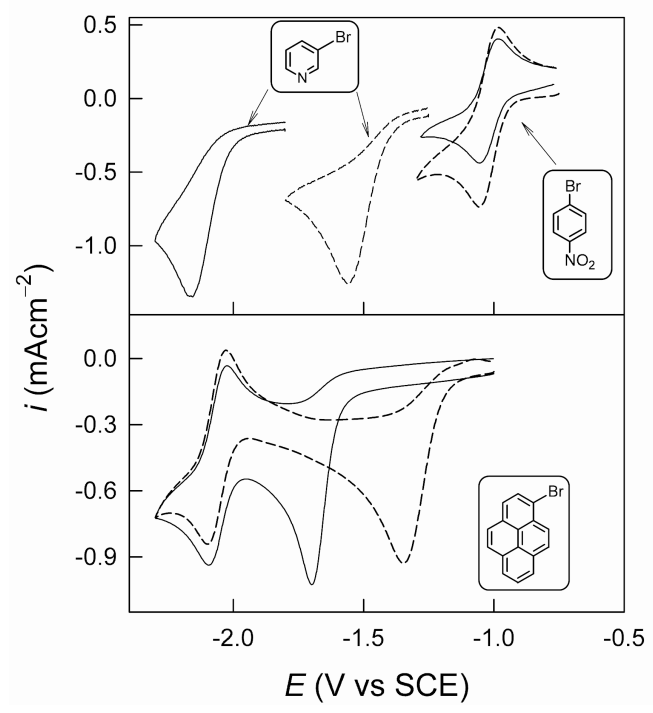

Fig. 2. Cyclic voltammetry of 4-bromonitrobenzene $\mathbf{7 b}$, bromopyridine $\mathbf{5 b}$ and bromopyrene $\mathbf{8}$ $(0.75 \mathrm{mM})$ in $\mathrm{CH}_{3} \mathrm{CN}+0.1 \mathrm{M} \mathrm{Et}_{4} \mathrm{NBF}_{4}$ recorded at $0.2 \mathrm{Vs}^{-1}$ at $\mathrm{GC}$ (full lines) and $\mathrm{Ag}$ (dashed lines).

The voltammetric behaviour of the nitro derivative $\mathbf{6 b}$ is similar to that of the corresponding chloride 6a; the first reduction step involves only one electron and is both chemically and electrochemically reversible. Since this reduction step is centred on the nitro group, no catalytic effect is observed at the Ag electrode. 


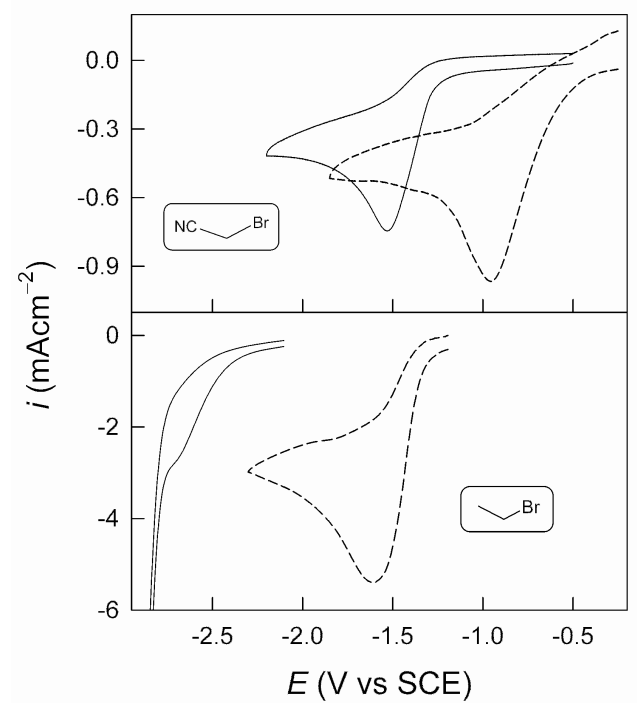

Fig. 3. Cyclic voltammetry of bromoacetonitrile $\mathbf{3 b}(0.75 \mathrm{mM})$ and bromoethane $\mathbf{1 b}(1 \mathrm{mM}$ at GC and $3 \mathrm{mM} \mathrm{Ag}$ ) in $\mathrm{CH}_{3} \mathrm{CN}+0.1 \mathrm{M} \mathrm{Et}_{4} \mathrm{NBF}_{4}$ recorded at $0.2 \mathrm{Vs}^{-1}$ at $\mathrm{GC}$ (full lines) and $\mathrm{Ag}$ (dashed lines).

Each of the aliphatic bromides 1a, 2a, 3b and $\mathbf{4 b}$ exhibit both at GC and Ag electrodes a single, irreversible reduction peak. These compounds undergo a concerted DET and, as expected, the transfer coefficients calculated at both electrodes are significantly smaller than 0.5 . Remarkable electrocatalytic effects are observed for the reduction of these compounds at $\mathrm{Ag}$; as compared to $\mathrm{GC}, E_{\mathrm{p}}$ at Ag positively shifts by $0.59-0.97 \mathrm{~V}$.

The voltammetric analysis of compounds $\mathbf{5 b}, \mathbf{6 b}$ and $\mathbf{8}$ give contrasting results at the two electrodes. At GC, the average values of $\alpha$ calculated for $\mathbf{5 b}, \mathbf{6 b}$ and $\mathbf{8}$ are $0.59,0.42$ and 0.76 , respectively. Although $\alpha$ of $\mathbf{6 b}$ is smaller than 0.5 , the DET mechanism of all these bromides can be fairly assigned as stepwise. Regarding Ag, the values of $\alpha$ calculated from $\partial E_{\mathrm{p}} / \partial \log v$ are much smaller than 0.5 and this may be taken to be indicative of a concerted DET mechanism. The silver electrode shows good catalytic activities also for these compounds. 


\subsection{Iodides}

All investigated iodides exhibit irreversible reduction peaks, with peak potentials strongly depending on the cathodic material (Fig. 4). The transfer coefficients calculated for all iodides at both electrodes are in the range from 0.36 to 0.43 . Although these values are smaller than 0.5 , indicating that the process is kinetically controlled by the ET, they do not allow unequivocal assignment of the DET mechanism. In analogy with chlorides and bromides, which have been more extensively investigated $[3,33,34]$, we may assume a concerted DET mechanism for the aliphatic iodides $\mathbf{1 b}, \mathbf{2} \mathbf{b}$ and $\mathbf{4 c}$ at both electrodes. As to the aromatic compound $\mathbf{6 c}$, DET of iodobenzenes is reported to be a borderline between concerted and stepwise mechanisms, with the possibility of a passage from one mechanism to the other, depending on the reaction conditions [35]. Therefore, we may assume a borderline situation for 4-iodotoluene. Interestingly, Ag exhibits extraordinary electrocatalytic activities for all four compounds, even if the catalytic effect shows a strong

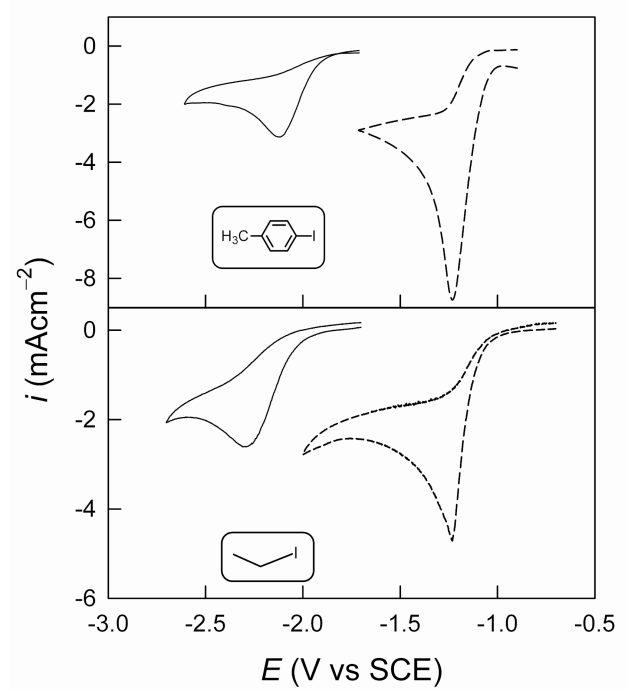

Fig. 4. Cyclic voltammetry of iodotoluene and iodoethane $(3 \mathrm{mM})$ in $\mathrm{CH}_{3} \mathrm{CN}+0.1 \mathrm{M} \mathrm{Et}_{4} \mathrm{NBF}_{4}$ recorded at $0.2 \mathrm{Vs}^{-1}$ at GC (full lines) and $\mathrm{Ag}$ (dashed lines). 
dependence on the molecular structure of the substrate. In fact, the positive shifts of $E_{\mathrm{p}}$ with respect to $\mathrm{GC}$ span a quite wide range from $0.58 \mathrm{~V}$ for $\mathbf{2 b}$ to $1.06 \mathrm{~V}$ for $\mathbf{1 b}$.

\section{Discussion}

The comparison between the electrochemical behaviour of compounds 1-8 at GC and Ag gives some interesting indications on the role of the electron transfer mechanism on the electrocatalytic activity of silver. Analysis of the data reported in Tables 1 and 2 allows identification of three main different types of behaviour.

\subsection{Nitro compounds}

The mechanism of the electrochemical reduction of nitro compounds has been widely investigated and is well understood [32]. The ability of the nitro group to accommodate the incoming electron, thanks to the strong delocalisation of the $\pi^{*}$ orbital, makes this group the host for, at least, the first electron uptake, independently of the structure of the compound. Thus, it is a general finding that the first reduction of nitro compounds corresponds to a reversible one reduction process, with the formation of a very stable radical anion.

As already found the electrocatalytic activity of silver and other catalytic materials $[20,21]$ is insignificant in the case of reversible ETs. For example, the reduction of aromatic hydrocarbons, such as anthracene, phenanthrene, etc., is practically indifferent to the cathodic material. Interactions between the cathodic surface and the $\pi^{*}$ orbital are practically absent and the process is generally a typical outer sphere ET.

The two investigated nitro halides, $\mathbf{7 a}, \mathbf{b}$, give similar voltammetric responses, typical of nitrobenzenes. The first voltammetric peak corresponds to a reversible one electron reduction (see Figs. 1 and 2) with the formation of a stable radical anion. This process is completely unaffected by 
the cathodic material employed: the same voltammetric response, typical of a reversible ET, has been obtained both at GC and Ag electrodes. The peak potentials are identical and practically independent of the scan rate; the peak widths are comparable and are very close to the theoretical value of $56.5 \mathrm{mV}$; the ratio $I_{\mathrm{pa}} / I_{\mathrm{pc}}=1$. All these findings confirm that the ET involves the nitro group which is very little influenced by the halogen atom in the ring.

The peak potentials, or $E_{1 / 2}$ for bromo and chloro nitrobenzene are very similar, indicating a very poor influence of the halogen atom on the reduction process. This is in line with the above consideration that the nitro group is the centre of the reduction process and the halogen atom exerts only a typical substituent effect. In this context, $\mathrm{Br}$ is slightly more electron withdrawing than $\mathrm{Cl}$ (their $\sigma^{-}$values are 0.25 and 0.19 , respectively [36]) and, indeed, the reduction potential of the bromo derivative is slightly more positive than that of the chloro derivative.

The voltammetric responses show that the reversible ET to the nitro group is diffusion controlled. This means that the intramolecular electron transfer, from the $\pi^{*}$ orbital of the nitro group to the $\sigma^{*}$ carbon-halogen orbital is very slow and occurs far from the electrode surface, which does not exert any influence on this intramolecular ET and the consequent carbon-halogen bond breaking.

\subsection{Aliphatic halides}

The electrochemical reduction of aliphatic halides occurs via a concerted ET. The absence of an energetically accessible $\pi^{*}$ orbital and the very high energy of the $\sigma^{*}$ carbon-halogen orbital makes unfavourable the formation of the corresponding radical anion. Thus, the only viable way of accommodating the incoming electron is elongation of the carbon-halogen bond to lower the energy of the $\sigma^{*}$ orbital which, upon injection of the electron, goes on to the complete breaking. In these cases, the dynamics of the heterogeneous ET may be sensitive to the nature of the cathodic surface. Thus, in the case of cathodic materials possessing catalytic activities such as $\mathrm{Ag}$ [13-16, 19-22], $\mathrm{Cu}$ 
$[21,23,24]$ and $\operatorname{Pd}[21,25,26]$, there are significant interactions between the electrode surface and, possibly the reagents, products, and mainly the activated complex [21], resulting in a lowering of the activation energy of the ET. As a consequence, the reduction process occurs at more positive potentials (less overpotential is required) with respect to a non-catalytic electrode.

For all of the investigated aliphatic halides $\mathbf{1 a}, \mathbf{b}, \mathbf{2 a}, \mathbf{b}, \mathbf{3 a}, \mathbf{b}$ and $\mathbf{4 a}, \mathbf{b}, \mathbf{c}$, the data reported in Tables 1 and 2 are in good agreement with the concerted DET mechanism. In particular, the electron transfer coefficient $\alpha$, at $\mathrm{GC}$, is generally lower than 0.35 . This is true also at $\mathrm{Ag}$, even if in some cases the process is not completely diffusion controlled because of the interactions with the electrode surface. Very strong catalysis has been observed for all these halides, as shown by the last column of Table 2: a positive shift of the reduction peak of several hundreds of millivolts is always observed.

Examination of the reported $\Delta E_{\mathrm{p}}$ suggests some further considerations. At GC the reduction potentials of RX are in line with the well known behaviour: $\mathrm{I}>\mathrm{Br}>\mathrm{Cl}$. This is well evidenced by compounds $\mathbf{4 a , b , c}$, which allow a comparison of all three halides, but also by all other couples of halides, $\mathrm{Cl}$ versus $\mathrm{Br}$ or $\mathrm{Br}$ versus I. This finding is strongly related to the bond dissociation energy (BDE), which increases in the opposite direction $(\mathrm{I}<\mathrm{Br}<\mathrm{Cl}$ ), because this parameter influences both the thermodynamics $\left(E_{\mathrm{RX} / \mathrm{R}^{\bullet}, \mathrm{X}^{-}}^{\oplus}\right.$ of the concerted DET depends on BDE [3]) and the kinetics (the intrinsic barrier and, consequently, the activation energy contain a contribution equal to BDE/4 [8]) of the process. This is true also at $\mathrm{Ag}$, indicating that BDE always plays a primary role. In this case, however, surface interactions with reagents, products and activated complexes are also important and therefore the electrocatalytic activity has not always the same sequence.

In general the electrocatalytic effect is expected to be related to the difficulty of reduction of RX or simply to the BDE of the carbon-halogen bond. This means the greater the overpotential (more difficult reduction), the greater the expected electrocatalytic effect. On the other hand, adsorption of the products, in particular the halide ions, provides a thermodynamic contribution to the electrocatalysis. It has recently been shown that adsorption of $\mathrm{Br}^{-}$and $\mathrm{I}^{-}$at $\mathrm{Ag}$ is relevant at 
potentials greater than -1.33 and $-1.50 \mathrm{~V}$ vs SCE, respectively [37]. The desorption potential of $\mathrm{Cl}^{-}$ is less defined, but is certainly more positive than that of $\mathrm{Br}^{-}$. Now, in the case of compounds $\mathbf{1 a}, \mathbf{b}$, the Ag electrode exhibits higher catalytic effects for iodoethane than for bromoethane, whereas the opposite is true for compounds 2a,b. Comparing $\mathrm{Cl}$ with $\mathrm{Br}$, there is higher catalysis for the chloride than for the bromide in the case compounds 3a,b, whereas the opposite is true for compounds 4a,b. A comparison of the whole set of halogen atoms is possible only for the benzyl halides $\mathbf{4 a , b , c}$, in which case the highest electrocatalytic effect is found for the bromide, similar electrocatalytic effects being observed for the chloride and iodide.

A survey of the reduction potentials reported in Table 2 and the desorption potentials of $\mathrm{Br}^{-}$ and $\Gamma$ reveals that thermodynamic contribution due to specific adsorption of halide ions on $\mathrm{Ag}$ could be important only in the case of $\mathbf{1 b}, \mathbf{2 b}, \mathbf{3 b}, \mathbf{4 b}, \mathbf{4 c}$ and $\mathbf{6 c}$. On the other hand, the kinetic effect is always present and is expected to increase with increasing BDE. It appears that kinetic contribution prevails for compounds $\mathbf{2}$ and $\mathbf{3}$, whereas a strong thermodynamic contribution due to specific adsorption of $\Gamma^{-}$causes an inversion of the sequence for compounds $\mathbf{1 a , b}$. In fact, at the reduction potential of $\mathbf{1 a}$ at $\mathrm{Ag}, \mathrm{Br}^{-}$is not adsorbed at the electrode, whereas adsorption of $\mathrm{I}^{-}$plays a crucial role in the electrocatalytic reduction of $\mathbf{1 b}$, which is reduced at potentials where the halide ion is strongly adsorbed. Also the electrocatalytic trend observed for compounds $\mathbf{4 a , b , c}$ can be rationalised by considering both kinetic and thermodynamic contributions. In particular, while the kinetic contribution of $\mathrm{Ag}$ surface is always present with the normal sequence $\mathrm{I}<\mathrm{Br}<\mathrm{Cl}$, the thermodynamic contribution due to adsorption is present only in the case of $\mathrm{Br}^{-}$and $\mathrm{I}^{-}$, with the usual sequence $\mathrm{I}>\mathrm{Br}$, whereas it is insignificant for the $\mathrm{Cl}^{-}$. Thus, benzyl chloride has only the BDE contribution, whereas both the bromide and the iodide have two contributions and because of this the catalytic effect observed for $\mathbf{4 a}$ is smaller than that of the bromide $\mathbf{4 b}$. Comparable electrocatalytic effects are observed for $\mathbf{4 a}$ and $\mathbf{4 c}$, even though the latter has two contributions. Possibly, the additional thermodynamic contribution of the iodide is compensated by the considerably higher kinetic effect of the chloride; the bond dissociation energies of $\mathbf{4 a}, \mathbf{4 b}$ and $\mathbf{4 c}$ 
are 309,235 and $187 \mathrm{kJmol}^{-1}$, respectively [38]. Comparing $\mathbf{4 b}$ and $\mathbf{4 c}$, since both compounds have an important thermodynamic contribution, the role of the BDE prevails in the overall electroatalytic effect, resulting in a higher catalysis for the bromide.

\subsection{Aromatic halides}

The electrochemical behaviour of the aromatic halides is more complex than that of the aliphatic halides and is strongly influenced by the nature of halogen atom. The voltammetric responses of $\mathbf{5 a}, \mathbf{b}, \mathbf{6} \mathbf{a}, \mathbf{b}, \mathbf{c}$ and $\mathbf{8}$ at GC are coherent with the stepwise DET mechanism. However, some differences can be outlined: the electron transfer coefficient $\alpha$ is quite higher than 0.5 for compounds $\mathbf{5 a}, \mathbf{b}, \mathbf{6} \mathbf{a}$ and $\mathbf{8}$, whereas it is slightly lower than 0.5 for compounds $\mathbf{6 b}, \mathbf{c}$. This finding seems to indicate that stepwise DET is the more preferred mechanism and this preferrance increases with increasing accessibility of the $\pi^{*}$ LUMO, i.e with lowering of its energy. At the same time, increasing the BDE increases the activation free energy of the concerted mechanism, thus favouring the stepwise route. The role of the $\pi^{*}$ orbital energy becomes well evident by a close examination of both the chloride and bromide series. The trends of $\pi^{*}$ energies are $\mathbf{5 a}<\mathbf{6 a}$ for the chlorides and $\mathbf{8}<\mathbf{5 b}<\mathbf{6} \mathbf{b}$ for the bromides $[39,40]$. This trend is exactly the opposite of that found for the $\alpha$ values. On the other hand, if we reasonably assume that the effect of the halogen atom on the energy of the $\pi^{*}$ orbital of the aromatic system is negligible, $\alpha$ is found to be related to the BDE; roughly the two parameters show the same trend: $\mathbf{5 a}>\mathbf{5 b}, \mathbf{6} \mathbf{a}>\mathbf{6 b}>\mathbf{6} \mathbf{c}$.

The behaviour of chlorides 5a and $\mathbf{6 a}$ is in perfect agreement with the general finding [20,21] for chlorides: in the case of stepwise ET no catalysis is displayed by Ag.

Quite different is the situation for bromides, which, at least at GC, undergo stepwise reductive cleavages. In these cases, Ag always displays electrocatalysis, even though the degree of catalysis strongly depends on the molecular structure and appears to be related to the stepwise tendency of 
the DET. In the case of bromopyrene $\mathbf{8}$, the highly delocalised $\pi^{*}$ orbital in which the incoming electron is accommodated, makes the stepwise mechanism more favourable than the concerted DET and, consequently, electrocatalysis is less significant. On the contrary, a very high electrocatalytic effect is observed for bromotoluene $\mathbf{6 b}$ in which the $\pi^{*}$ orbital is less delocalised with respect to $\mathbf{8}$ and hence the stepwise mechanism is less favourable. In this case, also a change of mechanism could occur at Ag, which would add a further thermodynamic contribution because of the more positive $E^{\Theta}$ for the concerted mechanism with respect to the stepwise one.

The electrocatalytic effect is very high also for the iodide $\mathbf{6 c}$. In this case, there is undoubtedly an important thermodynamic contribution due to the specific adsorption of $\mathrm{I}^{-}$at $\mathrm{Ag}$. It is worth noting, however, that the DET mechanism at the non catalytic electrode is in a borderline situation and it is highly likely to shift to the concerted limiting situation as the driving force of the reaction decreases owing to the electrocatalytic effect of $\mathrm{Ag}$.

\section{Conclusions}

The electrocatalytic activity of silver towards the reduction of organic halides is strongly affected by the DET mechanism. Remarkable electrocatalysis is always displayed in the case of concerted DET, independently of the nature of the halogen atom, even if the extent of catalysis may be affected by the possibility of adsorption of halide anions when the reduction potential at $\mathrm{Ag}$ is not so negative that electrostatic repulsions prevent $\mathrm{X}^{-}$adsorption.

In the case of stepwise DET, the situation is less univocal. If the centre of the ET is completely circumscribed to the host group, which does not interact with the halogen atom, as in the case of the nitro group, no electrocatalysis is displayed by $\mathrm{Ag}$, independently of the type of the halogen atom.

Dissociative ET to aromatic chlorides follows a stepwise mechanism owing to the very high energy level of the carbon-chlorine $\sigma^{*}$ orbital. In these cases, as already reported in the literature, 
no catalysis is displayed by $\mathrm{Ag}$ (as well as by other cathodic materials). Thus, for chlorides electrocatalysis is clearly discriminated by the DET mechanism. Conversely, there is no clear-cut discrimination of catalysis based on the DET mechanism in the case of aromatic bromides. In fact, Ag exhibits good electrocatalytic activities for all of the investigated aromatic bromides, which undergo stepwise DET at a non-catalytic electrode such as GC. A first indication of a possible correlation between the electrocatalytic effect and the dynamics of the DET seems to be apparent from the reported data: the more pronounced the stepwise mechanism, the lower the electrocatalytic effect. More extensive investigations are required to better develop this possible correlation, taking into consideration also the possibility of a mechanism change from stepwise to concerted.

In the case of the only aromatic iodide investigated, a remarkable electrocatalytic effect was observed. In this case the DET mechanism is borderline at GC and probably becomes concerted at $\mathrm{Ag}$ with significant thermodynamic advantage. In addition, adsorption of the iodide ion at $\mathrm{Ag}$ provides a further thermodynamic advantage, significantly contributing to the electrocatalysis.

\section{Acknowledgements}

This work was financially supported by the Ministero dell'Istruzione, dell'Università e della Ricerca.

\section{References}

[ 1] Hawley MD (1980) In: Bard AJ, Lund H (eds.) Encyclopedia of Electrochemistry of the Elements, vol. XIV, Marcel Dekker, New York, (Chapter 1).

[ 2] Peters DG (2001) In: Lund H, Hammerich O (eds.) Organic Electrochemistry, fourth ed., Marcel Dekker, New York, p. 341. 
[ 3] Savéant J-M (2006) Elements of Molecular and Biomolecular Electrochemistry, Wiley-

Interscience, New York.

[ 4] Casanova J, Reddy VP (1995) In: Patai S, Rappoport Z (eds.) The Chemistry of Halides, Pseudohalides and Azides, John Wiley \& Sons, New York, p. 1003.

[ 5] Liu Z, Betterton EA, Arnold RG (2000) Environ Sci Technol 34:804.

[ 6] Sonoyama N, Ezaki K, Sakata T (2001) Adv Environ Res 6:1.

[ 7] Rondinini S, Vertova A (2004) Electrochim Acta 49:4035.

[ 8] Savéant J-M (1987) J Am Chem Soc 109:6788.

[ 9] Rondinini S Mussini PR Muttini P Sello G (2001) Electrochim Acta 46:3245.

[10] Isse AA, Gennaro A (2002) Chem Commun 2798.

[11] Ardizzone S, Cappelletti G Doubova LM, Mussini PR, Passeri SM, Rondinini S (2003) Electrochim Acta 48:3789.

[12] Mussini PR, Ardizzone S, Cappelletti G, Doubova LM, Longhi M, Rondinini S (2003) J Electroanal Chem 552:213.

[13] Isse AA, De Giusti A, Gennaro A, Falciola L, Mussini PR (2006) Electrochim Acta 51:4956.

[14] Isse AA, De Giusti A, Gennaro A (2006) Tetrahedron Letters 47:7735.

[15] Bellomunno C, Bonanomi D, Falciola L, Longhi M, Mussini PR, Doubova LM, Di Silvestro G (2005) Electrochim Acta 50:2331.

[16] Gennaro A, Sánchez-Sánchez CM, Isse AA, Montiel V (2004) Electrochem Commun 6:627.

[17] Isse AA, Ferlin MG, Gennaro A, (2005) J. Electroanal. Chem. 581:38.

[18] Scialdone O, Galia A, Errante G, Isse AA, Gennaro A, Filardo G (2008) Electrochim Acta, $53: 2514$.

[19] Falciola L, Gennaro A, Isse AA, Mussini PR, Rossi M (2006) J Electroanal Chem 593:47.

[20] Isse AA, Falciola L, Mussini PR, Gennaro A (2006) Chem Commun 344.

[21] Isse AA, Gottardello S, Durante C, Gennaro A (2008) Phys Chem Chem Phys 10:2409.
Formattato: Inglese (Gran Bretagna)

Formattato: Italiano (Italia) 
[22] Isse AA, Gottardello S, Maccato C, Gennaro A (2006) Electrochem Commun 8:1707.

[23] Fedurco M, Sartoretti J, Augustynski J (2001) Langmuir 17:2380.

[24] Simonet J (2005) J Electroanal Chem 583:34 .

[25] Poizot P, Laffont-Dantras L, Simonet J (2008) J Electroanal Chem 622:204.

[26] Poizot P, Laffont-Dantras L, Simonet J (2008) Platinum Metals Rev. 52:84.

[27] Ardizzone S, Cappelleti G, Doubova LM, Mussini PR, Rondinini S (2002) Russ J Electrochem 39:182.

[28] Bard AJ, Faulkner LR (2001) Electrochemical Methods, 2nd edn. John Wiley \& Sons, New York.

[29] Andrieux CP, Blocman C, Dumas-Bouchiat J-M, Savéant JM (1979) J Am Chem Soc Formattato: Inglese (Gran Bretagna) $101: 3431$

[30] Andrieux CP, Savéant JM, Zann D (1984) Nouv J Chim 8:107.

[31] Altomonte S, Falciola L, Mussini PR, Trasatti S, Gennaro A, Isse AA (2008) Russ J Electrochem 44:104.

[32] Kraiya C, Singh P, Evans DH (2004) J Electroanal Chem 563:203.

[33] Andrieux CP, Gallardo I, Savéant J-M, Su KB (1986) J Am Chem Soc 108:638.

[34] Cardinale A, Isse AA, Gennaro A, Robert M, Savéant J-M (2002) J Am Chem Soc 124:13533.

[35] Pause L, Robert M, Savéant J-M (1999) J Am Chem Soc 121:7158.

[36] Hansch C, Leo A, Taft WR (1991) Chem Rev 91:165.

Formattato: Italiano (Italia)

[37] Falciola L, Mussini PR, Trasatti S, Doubova LM (2006) J Electroanal Chem 593:185.

[38] NIST Chemistry Webbook, available on line at http://webbook.nist.gov/chemistry/.

[39] Pierini AB, Vera DMA (2003) J Org Chem 68:9191.

[40] Krygowski TM, Stępień BT, Cyrański MK, Ejsmont K (2005) J Phys Org Chem 18:886. 